\title{
ePatienten: die Weisheit der Masse
}

Den ePatienten und dessen Möglichkeiten der Partizipation und Kollaboration in einem digitalen Gesundheitssystem beleuchtet Dr. cand. Alexander Schachinger in seinem Inspirationsreferat am eHealthCare.ch-Kongress. Im deutschsprachigen Raum gilt der Berliner Berater und Forscher als der Botschafter für Health 2.0 - jenes zunehmend partizipative Gesundheitssystem, in dem «der Patient sich digital emanzipiert», sich in sozialen Netzwerken austauscht und aktiv Daten für die Forschung und seine personalisierte Gesundheitsversorgung bereitstellt. Die Informationshoheit von Ärzten wird dadurch relativiert und ergänzt, sagt Schachinger.

Interview:

Armin Scheuer,

HealthTech Wire*

\footnotetext{
${ }^{*}$ HealthTech Wire ist der Nachrichtendienst des Kongresses eHealthCare.ch. Weitere Informationen unter www.healthtechwire.ch
}

Literatur zum Thema digitales Gesundheitssystem findet sich im Internet unter www.saez.ch

$\rightarrow$ Aktuelle Nummer oder

$\rightarrow$ Archiv $\rightarrow 2010 \rightarrow 35$

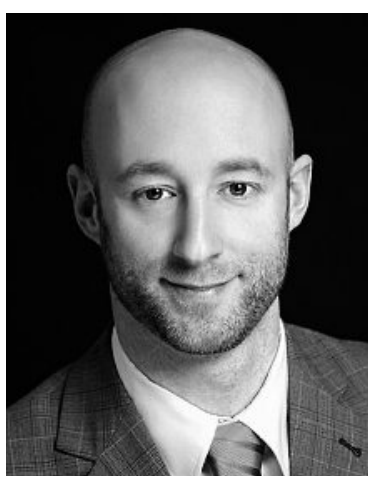

Alexander Schachinger: «Der medizinische Nutzen von Onlinenetzwerken ist zunehmend spürbar.»

Herr Schachinger, haben Sie digitale Gesundheitsapplikationen?

Alexander Schachinger: Nein. Ich bin gesund und habe keinen direkten Bedarf. Allerdings sollte ein digitaler und personalisierter Status Quo im Sinne der Primärprävention meine Krankenversicherung eigentlich interessieren. Damit Patienten digitale Gesundheitsleistungen annehmen, muss ein hoher Leidensdruck vorhanden sein - dies gilt vor allem bei chronischen und psychischen Erkrankungen, die im Alter von 20 bis 60 Jahren auftreten. Bluthochdruck, koronare Herzerkrankungen oder Osteoporose hingegen sind nicht so gut online therapierbar, weil der Leidensdruck nicht gross genug ist.

Wie kann Health 2.0 die Versorgung verbessern, und was verspricht sich diese neue Branche davon?

Die Entwicklung des Internets von einem reinen Kanal hin zu einem Netzwerk und einer Produktionsplattform ergibt völlig neue Ansätze digitaler Gesundheitsdienste. Solche Health-2.0-Anwendungen ermöglichen Lösungen für ein kohärentes, personalisiertes Chroniker-Management. Patienten lernen voneinander im Umgang mit Erkrankungen, erstellen Inhalte gemeinsam und helfen sich massiv gegenseitig. Das ist die Weisheit der Massen, das Wikipedia-Prinzip. Dadurch werden die Angaben einzelner Nutzer zu einem objektiven Ideal nivelliert. Ein Artikel auf Wikipedia ist nachweislich qualitativ identisch mit einem Artikel der Encyclopaedia Britannica.

Dieses Prinzip funktioniert jedoch erst auf einer quantifizierten und aggregierten Datenebene. Auf www.patientslikeme.com sammeln beispielsweise an die 60000 aktive Patienten medikamentöse Nebenund Verträglichkeitswirkungen. Diese ergeben in ersten Ansätzen pharmazeutische Forschungsqualität und Ergänzungen hierfür. Man erfährt so direkt von den Patienten, welche Therapien den besten Erfolg erzielen. Erste Medikamentenhersteller wie z.B. Novartis und UCB Pharma kooperieren daher bereits mit diesen Portalen.

November 2007: In der Amyotrophe-LateralskleroseCommunity bei dem von Ihnen erwähnten Patientennetzwerk vereinen sich mehrere Mitglieder ad hoc zu einer Testgruppe: Die Einnahme von Lithium soll den Krankheitsverlauf signifikant verlangsamen, heisst es; dies habe angeblich eine Studie aus Italien bewiesen. Was halten Sie von dieser Art der Partizipation?

Das mag bedenklich sein, ist aber nicht steuerbar. Digitale Netzwerke reduzieren grundsätzlich massiv die Transaktionskosten für Gruppenkoordination weltweit. Wenn die eine Plattform solche Selbsttests verbietet, findet sich eine andere. Man muss aber auch die Patienten verstehen: Sie haben chronische Erkrankungen, bei denen häufig der aktuelle Stand der Forschung nicht ausreicht. Um es mit den Worten des global bekanntesten E-Patienten Dave deBronkart zu sagen: «Eine Betroffenheit ändert alles.» Da machen Sie auch Lithiumtests.

Trotzdem ist der medizinische Nutzen von Onlinenetzwerken zunehmend spürbar. Der Umgang mit der eigenen Erkrankung, die Compliance und die Aufgeklärtheit der Patienten verbessern sich erwiesenermassen. Das Vertrauen im Gespräch mit «patients like me» 
ist höher als im Arztgespräch. Das hat auch meine Umfrage in Deutschland bestätigt.

\section{Eine Folge des Zeitmangels der Ärzte?}

Online-Aktivitäten von Patienten versuchen grundsätzlich, die persönlichen und fachlichen Versorgungsschwächen eines Gesundheitssystems zu kompensieren, beispielsweise die Informationsasymmetrie. Patienten wünschen sich heute von Ärzten, die ja immer noch Vertrauenspersonen erster Güte sind, Empfehlungen, wo sie sich selbst online informieren können. Schliesslich werden die digitalen Gesundheitsangebote von tendenziell aktiven, gebildeten Patientengruppen genutzt: Die Akademikerquote ist doppelt so hoch wie im Landesdurchschnitt.

\section{«Patienten lernen voneinander, erstellen Inhalte gemeinsam und helfen sich massiv gegenseitig»}

\begin{abstract}
Wie ist die Reaktion der Ärzte auf die neue Macht der Patienten?

Man hört manchmal schon, dass die neue Situation als ein Angriff auf ein Expertentum, auf ein Wissensmonopol, empfunden wird. Aber, eins ist klar: Der Arzt kann nicht ersetzt werden, wie der mittelalterliche Schreiber durch die Druckerpresse. Kein E-Patient kann sich selbst den Blinddarm entfernen. Aber ein Diabetiker ist 8700 Stunden im Jahr mit seiner Erkrankung konfrontiert, der Arzt sieht ihn hingegen nur 2 Stunden im Jahr - hier fehlt massives Aufklärungs- und Krankheits-Management. Um die Compliance, das Engagement und das Patientenvertrauen zu verbessern, gilt die Devise: die richtige Information zum richtigen Patienten zum richtigen Zeitpunkt. Hier können Online-Angebote den Arzt unterstützen. Eine Versorgung mit personalisierten, relevanten Informationen und Applikationen ist dadurch möglich. Dies ist ein Paradigmenwechsel hin zur gemeinsamen Entscheidung für die beste Gesundheitsversorgung.
\end{abstract}

Was bedeutet das konkret? Wie sollen sich Ärzte auf Patientenpartizipation einstellen?

Wir sind in einer Umbruchphase. Viele Gesundheitsangebote im Internet sind in der Entwicklungs- und Erprobungsphase. Es gibt aber bereits standardisierte, sinnvolle Modelle - diese entstehen leider ausserhalb des existierenden Gesundheitssystems. Die Frage ist, wie man beide Welten miteinander vernetzt.

10. Schweizerischer eHealthcare-Kongress, Mittwoch und Donnerstag, 22., 23. September 2010, GZI Seminar- und Kongresshotel, Nottwil LU An diesem Jubiläums-Kongress wird Dr. cand. Alexander Schachinger eine Inspiration zum Thema Health2.0 halten.

Die Schweizerische Ärztezeitung ist Medienpartner des Kongresses eHealthCare.ch. Das Konferenzprogramm und alle weiteren Informationen finden Sie online unter www.ehealthcare.ch.
In Dänemark ist seit Jahren die digitale Gesundheitsakte Realität. Der Patient und jeder behandelnde Arzt haben eine gemeinsame Datenbasis. Der Patient könnte nun über sein iPhone zusätzliche Daten zu seinen Sport- und Essgewohnheiten erfassen. Diese Datengrundlage könnte mit Patientenplattformen vernetzt werden. Zur Zeit gibt es aber zwischen diesen zwei Welten noch viele Barrieren.

Krankenhäuser und Leistungserbringer sollten zumindest online Standards definieren, um Partizipation und Interaktion zu ermöglichen. Patienten wünschen sich das. 80\% aller Internetsurfer sind zum Thema Gesundheit online unterwegs.

Wie kann ein Krankenhaus sich in dem neuen digitalen Gesundheitssystem positionieren?

Krankenhäuser müssen sich fragen, ob sie ihre Positionierung und Evaluation völlig dem Patienten überlassen wollen und das Handtuch in der Marketing- und Kommunikationskontrolle werfen, oder ob sie aktiv mit der neuen Situation umgehen - d. h. die Weisheit der Massen nutzen, um zuzuhören, zu verstehen, und neue Mehrwerte und Dienste darauf basierend anbieten. Der digitale Innovationskanon bietet viele klassische Marketing- und Kommunikationsmöglichkeiten, aber auch neue Serviceoptionen beim DiseaseManagement und in der Verbesserung der Compliance. Die Ansätze sind vielschichtig.

Thema Datenschutz - ich zitiere die Auffassung des führenden US-Patientenportals: "Zurzeit ist ein Grossteil der medizinischen Daten aufgrund von Datenschutzbestimmungen und urheberrechtlichem Taktieren unzugänglich. (...) Wenn Sie und tausende andere Patienten wie Sie Ihre Daten bereitstellen, öffnen Sie das Gesundheitssystem.» Den Patienten ist der Datenschutz egal. Sie haben einen klaren Leidensdruck, sie geben ihre Daten weiter und sind damit einverstanden, dass sie gesammelt werden, gerade für und im Sinne einer Weiterführung der Forschung. Der Schutz dieser Daten und die ausschliesslich anonymisierte Weiterverwendung in der Forschung müssten rechtlich gewährleistet sein.

Wie wird Health 2.0 im Jahr 2020 aussehen? Clayton Christensen beschreibt in seinem Buch «The Innovators Prescription» leider klar, dass das Gesundheitssystem aufgrund seiner Regulierung und festgefahrenen Partialinteressen nicht in der Lage ist, die digitalen Möglichkeiten aus sich heraus zu adaptieren. Krankenhäuser und Krankenkassen können sich nicht selbst aus ihren festgefahrenen IT-Strukturen herausarbeiten. Deshalb wird die Adaption von aussen geschehen, aktuell schon im Wesentlichen von privatwirtschaftlichen Anbietern aus der Software-, IT- und Medienbranche. Leider gibt es für die Krankenkassen diesbezüglich noch keinen grossen Handelsdruck, aus sich heraus diese Innovationen zu übernehmen. Die Krankenhäuser, Pharmaunternehmen oder Medizintechnikhersteller bewegen sich aber bereits deutlich in diese Richtung. 


\section{Ergänzung zum Interview mit Dr. cand. Alexander Schachinger}

\section{Literatur zum Thema digitales Gesundheitssystem:}

http://www.ncbi.nlm.nih.gov/pmc/articles/PMC2018833/?tool=pmcentrez

http://journal.lib.uoguelph.ca/index.php/perj/article/view/244/374

Christensen, Clayton M.: The Innovator's Prescription. A Disruptive Solution for Health Care. New York: McGraw-Hill 2009

Eysenbach, Gunther: Medicine 2.0. Social Networking, Collaboration, Participation, Apomediation, and Openness. Journal of Medical Internet Research, University of Toronto, Kanada, 2008;10(3):e22

Ferguson, Tom: e-Patients. How they can help us heal healthcare. New York: Association of Cancer Online Resources. White Paper der e-Patient Scholar Working Group,2007, online: http://e-patients.net/ (16. November 2009)

Fox, Susannah; Jones, Sidney: The Social Life of Health Information. Washington, USA: Pew Internet \& American Life Project 2009

Kemper, Donald W.; Mettler, Molly: Information Therapy. Prespribed Information as a Reimbursable Medical Service. Idaho: Healthwise Inc. 2002

Lasker, Judith: The Role of an Online Community for People With a Rare Disease: Content Analysis of Messages Posted on a Primary Biliary Cirrhosis Mailinglist. Toronto: Journal of Medical Internet Research, 2005, elektronische Resource: (J Med Internet Res 2005;7(1):e10) doi:10.2196/jmir.7.1.e10

Murero, Monica; Rice, Ronald E.: The Internet and Healthcare. Theory, Research and Practice. New Yersey: Lawrence Erlbaum Associates, Inc. 2006

Nettleton, Sarah: The Emergence of E-Scaped Medicine? Sociology, Thousand Oaks, USA, 2004; Heft 38/4, Seite 661-679

Sarasohn-Kahn, Jane: The Wisdom of Patients. Oakland: California Healthcare Foundation 2008

Sillence;Briggs;Harris;Fishwick: How do patients evaluate and make use of online health information? Social Science and Medicine, dio:10.1016, Article in Press, 2007

Tenderich, Amy: Reviewing Health Tools: A Community Matter. USA: Journal of Participatory Medicine, Launch Issue, Oktober 2009, elektronische Ressource: 1(1):e9

Wenger, Etienne: Community of Practice. Learning, Meaning and Identity. USA: Cambridge University Press. 17. Auflage 2008

Zerfass; Welker; Schmidt: Kommunikation, Partizipation und Wirkungen im Social Web.

Band 1 und 2. Köln: Herbert von Halem Verlag, 2008 